\title{
Implementación de tecnologías de información y administración del conocimiento en empresas consultoras
}

\author{
Danny Arévalo Avecillas \\ Universidad Católica de Santiago de Guayaquil \\ danny.arevalo@cu.ucsg.edu.ec \\ Carmen Padilla Lozano \\ Universidad Católica de Santiago de Guayaquil \\ carmen.padilla@cu.ucsg.edu.ec
}

Fecha de presentación: 19 de octubre de 2015 • Fecha de aceptación: 11 de agosto de 2016

Artículo de investigación 


\section{Resumen}

El artículo analiza empresas consultoras de Guayaquil que utilizan como medio de innovación la implementación de las tecnologías de la información en sus actividades diarias. La metodología es cualitativa y la herramienta utilizada una entrevista. El aporte de la investigación es la construcción de un mapa cognitivo de las TI para empresas que buscan mejorar su productividad mediante la implementación de tecnología.

Palabras clave: tecnologías de información (TI), empresas consultoras, innovación, administración del conocimiento, ventaja competitiva.

JEL: M1 Administración de empresas.

\section{Abstract}

The article analyzes consulting firms in Guayaquil, which use as a means of innovation, the implementation of information technologies in their daily activities. The methodology is qualitative; and the tool used an interview. The contribution of the research is the construction of a cognitive map of information technologies for companies that seek to improve their productivity through the implementation of technology.

Keywords: Information Technologies, Consulting Companies, Innovation, Information Management, Competitive Advantage.

JEL: M1 Business administration.

\section{Resumo}

O artigo analisa empresas de consultoria em Guayaquil, que utilizam como meio de inovação a implementação de tecnologias de informação em suas atividades diárias. A metodologia é qualitativa; e a ferramenta usava uma entrevista. O contributo da pesquisa é a construção de um mapa cognitivo das tecnologias da informação para as empresas que buscam melhorar sua produtividade através da implementação da tecnologia.

Palavras-chave: Tecnologias da informação (TI), empresas de consultoria, inovação, administração do conhecimento, vantagem competitiva.

JEL: M1 Administração de empresas. 


\section{Introducción}

cuador está conformado por distintos tipos de empresas, que van desde micro a grandes organizaciones. Según el Censo Nacional Económico de 2010 (INEC), más del 90\% de las actividades económicas son llevadas a cabo por microempresas que han aportado al crecimiento económico y sustentable del país.

Según la revista Ekos (2013), Ecuador presentó un escenario positivo en su desarrollo económico - para el año de investigación-, se encuentra en la posición número cuatro en relación a siete países de Latinoamérica y primera en relación con Centroamérica, cifra que se la obtuvo al comparar el Producto Interno Bruto (PIB) que generaron los países mencionados, según consta en la figura 1.

Figura 1

Desarrollo económico en la región

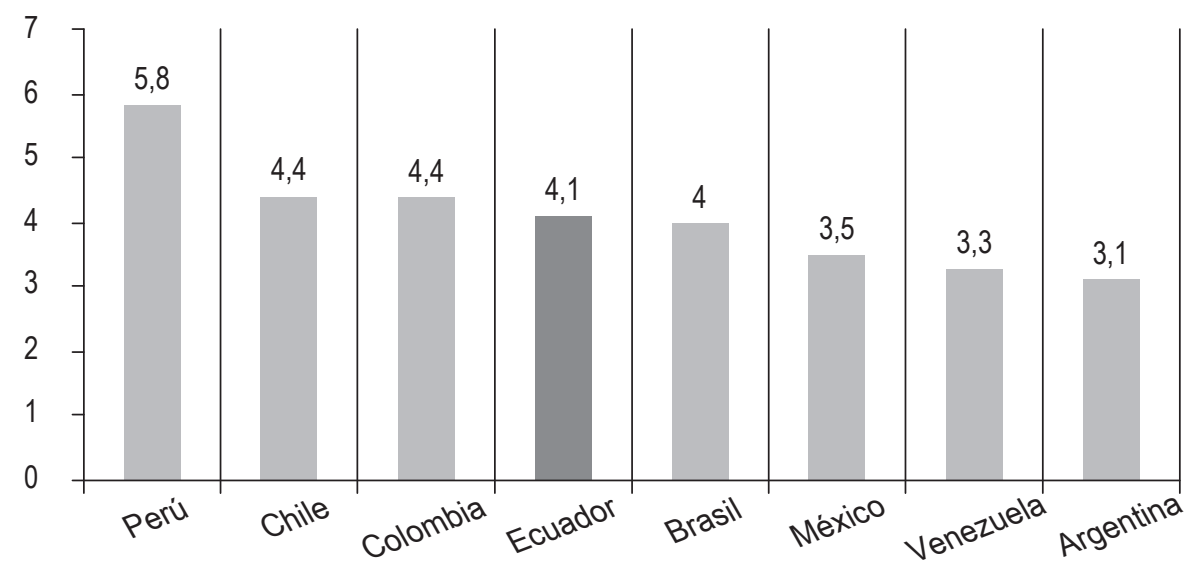

Fuente: adaptado de Ekos Negocios 2013.

Elaboración propia. 
La aparición de nuevos mercados y necesidades ha incrementado la demanda de empresas dedicadas a servicios de consultoría en áreas como: actividades jurídicas, arquitectura e ingeniería, investigación científica y desarrollo, consultorías de gestión empresarial, de investigación de mercado, publicidad, entre otras.

La actividad económica del Ecuador se encuentra distribuida en sus cuatro regiones: Costa, Sierra, Amazonía e Insular. El 95,29\% de la actividad económica está en la región litoral, con un 42,29\%, y en la región interandina con el 53\%, según consta en la figura 2 .

Figura 2

Actividad económica de Ecuador por región, 2010

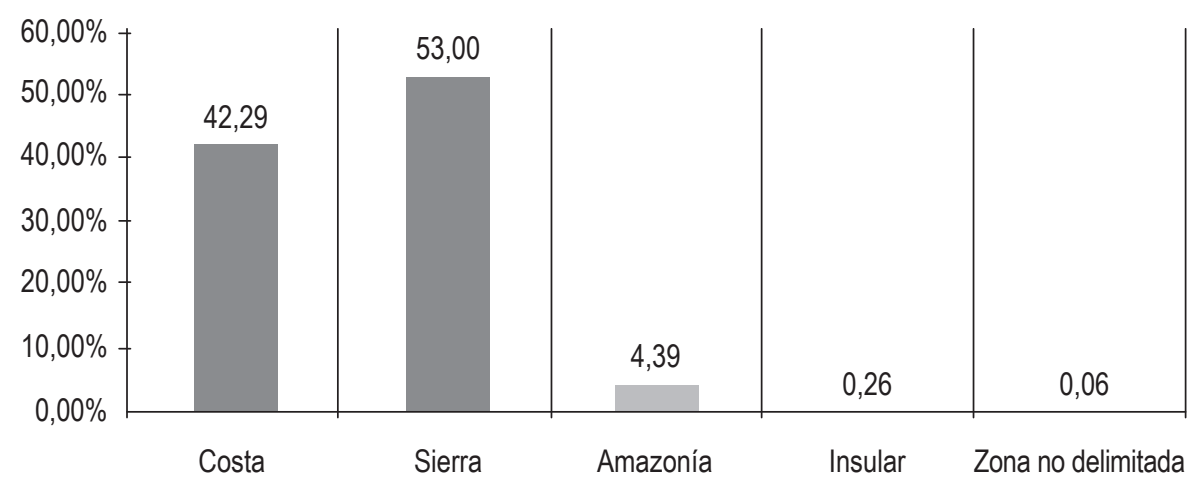

Fuente: adaptado de INEC 2014.

Elaboración propia.

Según el INEC (2014) existen 2.135 establecimientos relacionados con actividades profesionales, científicas y técnicas en la ciudad de Guayaquil. Las actividades profesionales, científicas y técnicas representan el 2,66\% a nivel nacional, destacando con un $54 \%$ el comercio al por mayor y menor, es decir, más de la mitad de las empresas ecuatorianas, ya sean pequeñas, medianas o grandes, se dedican a esta actividad.

La producción local se orienta hacia bienes de consumo no duradero o de consumo masivo; por citar un ejemplo, aproximadamente $39,3 \%$ de la producción industrial local se centra en la elaboración de alimentos y bebidas. 
Por otra parte, las actividades de servicios generan un aporte importante al producto interno bruto (PIB), ya que durante el 2012 las actividades profesionales, técnicas y administrativas (que incluye las actividades de consultoría y gestión) en conjunto aportaron con US \$ 4.888 millones al PIB ecuatoriano $(7,6 \%)$, lo que refleja una alta generación de valor agregado a nivel sectorial.

Según la Cuarta Revisión de la Clasificación Industrial Uniforme (CIIU) (INEI 2010), las actividades profesionales, científicas y técnicas son aquellas que requieren un nivel elevado de capacitación, y dentro de estas actividades se encuentra: a) actividades jurídicas y de contabilidad, b) actividades de oficinas principales y actividades de consultoría de gestión, c) actividades de arquitectura e ingeniería, d) investigación científica y desarrollo, e) publicidad y estudios de mercado, f) otras actividades profesionales, científicas y técnicas. En primera instancia, para la presente investigación se generó un proceso de análisis para determinar las características del estudio, es decir, hechos importantes e información relevante de las consultoras.

El Networked Readiness Index Ranking, realizado por el World Economic Forum (WEF 2014), que mide el nivel de aprovechamiento de las tecnologías de información (TI) de 148 países, ubicó a Ecuador en 2014 en el puesto 82 , subiendo nueve puntos en comparación con la posición número 91 de 2013. La revista Ekos (2014) citó al WEF y manifestó que un 10\% de penetración de banda ancha genera un incremento del $0,25 \%$ del PIB y que los países con mayor penetración de TI experimentan una productividad laboral de hasta siete veces mayor.

El estudio que Cimoli y Correa (2010) llevaron a cabo, con información de 46 países, para el período 1994-2003, estudia la permanencia de la brecha en el tiempo, si se ha ampliado o si se ha reducido, principalmente por la importante diferencia que hay entre la tecnología actual y la tecnología existente hace 10 años, tanto a nivel de hardware, software e infraestructura tecnológica, e incluso por temas de accesibilidad (precios) y disponibilidad.

Lopéz, Sepúlveda y Arenas $(2010,54)$ indican que:

Los servicios de consultoría se clasifican de múltiples maneras. Según la forma de intervención y el tipo de relación establecida entre consultor y cliente se han identificado tres tipos; modelo de compra de información o experiencia, modelo médico-paciente y la consultoría de procesos, en la cual el consultor orienta al cliente para que él mismo perciba su realidad empresarial. 


\section{Revisión de la literatura: uso de tecnologías de información en consultoras}

La economía moderna ha sido cada vez más dominada por los servicios; en América Latina los servicios representaron el 66\% del valor añadido en 2007, muy por encima del 49\% que se registró en 1977 (Francois y Hoekman 2010). Un ejemplo de servicios son las consultoras que son empresas compuestas por un conjunto de habilidades y capacidades para llevar a cabo sus estrategias comerciales (Allen y Meyer 1990), como necesidad desde el momento en que globalmente distintas empresas, en distintos sectores económicos, integran como parte de sus procesos o de su cadena productiva el uso de servicios informáticos, tecnológicos y de comunicaciones (Aral, Brynjolfsson y Wu 2012).

Finalmente, como herramienta en el momento en que una empresa en particular descubre que, indistintamente del sector en que se desempeñe, el uso de herramientas tecnológicas y de comunicación puede contribuir a obtener mejores resultados, incluso si el uso de las mismas no es indispensable para la operación de la empresa (Wade y Hulland 2004).

Por ejemplo, en la década del 80, la TI fue uno de los diferenciadores más importantes a nivel de los bancos (Nolan y McFarlan 1994). Empresas como American Airlines, Merrill-Lynch y Frito-Lay fueron ampliamente publicitadas por sus rendimientos superiores debido a sus programas implementados de TI (Nachum 1999). Según Devaraj y Kholi (2003), encontraron mejoras sustanciales en el rendimiento financiero en la industria de la salud como resultado de sus inversiones en TI. Sin embargo, el uso o implementación de TI depende del nivel de cultura organizacional de la empresa, debido a que esta depende, en primera instancia, de decisiones familiares (pequeñas y medianas empresas) y al retorno sobre la inversión a corto plazo que necesita ser cuantificado utilizando criterios de común aceptación, de los cuales se carece actualmente (Enríquez Martínez 2006).

El acceso a información de mercado evidentemente facilitaría estrategias de posicionamiento de productos y temas particulares como la gestión de valor de una marca, fidelidad, entre otros. Tal es el caso de la consultora de investigación de mercados radicada en Quito, Advance Consultora, que cada año elabora un Top of Mind para la revista Vistazo (2013). La empresa man- 
tiene la metodología del año 2012 con 8.130 encuestados en trece ciudades e indican que el Top of Mind es una construcción individual, dependiente de cada consumidor, de la publicidad, de la experiencia con la marca y de varios aspectos valorados por el consumidor (Arzola y Mejias 2010).

Otro ejemplo es el caso de Google Analytics, que en su página web se define como una herramienta que no solo permite medir ventas y conversaciones, sino que también ofrece información sobre cómo utilizan los visitantes los sitios webs de los clientes contratantes del servicio de Google, además de cómo han llegado a él y qué se puede hacer para que lo sigan utilizando.

\section{Innovación y las tecnologías de información para incrementar la competitividad}

Las empresas consultoras están compuestas por un conjunto de habilidades y capacidades para llevar a cabo sus estrategias comerciales; Kubr (2002) menciona que a los consultores se les pide integridad y competencia, con el fin de generar confianza, habilidad para los contactos, experiencia y conocimiento para resolver los temas planteados, capacidad de planificación, cumplimiento de entrega, control de costos, calidad y una adecuada imagen profesional (Bayo-Moriones, Billón y Lera-López 2013). El generar confianza es una condición necesaria en la relación con el cliente, por lo que una de las metas, antes de la firma de contrato, es conseguir que el prospecto de cliente confíe en él, demostrando sus capacidades técnicas para encontrar y solucionar problemas.

Varios autores están de acuerdo en que la confianza es una dimensión crítica en la relación cliente y consultor (Karantinou y Hogg 2001; Kubr 2002; Maister, Green y Galford 2000). La confianza debe ser desarrollada desde: a) una relación basada en la oferta de servicio, b) una relación basada en necesidades y c) una basada en la relación (Kubr 2002).

Normalmente se suele reconocer a la innovación como un componente que permite a las empresas ser competitivas o alcanzar una ventaja competitiva a largo plazo, mientras que, en un contexto macroeconómico, se la reconoce como un componente del crecimiento de un país. 
Schumpeter, citado por Freeman (1974) indicaba que:

La innovación hace posible que exista el crecimiento y el desarrollo socioeconómico, por medio de un efecto de retroalimentación. La innovación es primordial para que las naciones aumenten su riqueza y, además, permite al hombre cambiar su calidad de vida. Puede dar lugar no solo a una mayor cantidad de bienes, sino también a nuevos servicios. (Freeman 1974, 45).

Para el momento de elaboración del estudio de Darroch y McNaughton (2002), a pesar de que se reconocía la necesidad de una administración efectiva del conocimiento, no era posible identificar de manera exacta lo que representaba ser efectivo en dicha área.

La innovación se clasifica en tres tipos: a) incremental, b) orientada al consumidor, y c) radical y dependen del grado de tecnología que apliquen para incrementar su eficacia. La innovación se ha convertido en un fenómeno complejo de gestionar, puesto que se busca la administración correcta del conocimiento por medio de estrategias basadas en TI (Alavi y Leidner 2001).

El uso de TI genera eficacia en diversos aspectos. En educación, se encuentra que las nuevas TI promueven una visión del conocimiento y del aprendizaje (Bartolomé 1995) e implica que el tiempo se reduzca a medida que corrigen fallas a través de la retroalimentación. Además, eliminan las barreras espacio-temporales y condicionan la enseñanza presencial y a distancia (Cañellas 2006). En la medicina, el uso de TI para documentar diferentes aspectos en pacientes con traumas psicológicos permite abordar de forma eficiente y eficaz dichos tratamientos (Botella Arbona y otros 2010).

En cuanto al ámbito empresarial, en una muestra de 400 pequeñas y medianas empresas de Aguascalientes en México encontró un impacto positivo por parte de las TI que generan una ventaja competitiva (Maldonado Guzmán y otros 2010), corroborando lo dicho por Tung y Rieck (2005) quienes exponen que las TI permiten mejorar toda actividad administrativa de las empresas, especialmente en las pymes. Por lo tanto, es claro el papel de las TI en la eficacia y la eficiencia (Kantis 1996).

El uso de TI no es suficiente para mantener los aspectos antes nombrados, ya que también es necesario un almacenamiento suficiente y accesible para la gestión de la información, sobre todo en las consultoras que tienen personal de campo distribuido en muchos lugares. La revista Ekos (2016) 
expone las siguientes ventajas del uso del cloud en las empresas: a) Agilidad para integrar recursos o servicios en cuestión de minutos; b) flexibilidad para aumentar o reducir recursos en función de las necesidades empresariales; c) escalabilidad para adquirir mayor cantidad de recursos a medida que la empresa crece; d) movilidad para acceder a la "nube" desde cualquier lugar y en cualquier momento, a través de una conexión a internet (76).

De esta manera es evidente que el uso de la nube es vital para la comunicación eficiente y eficaz en las empresas.

El uso de Big Data y Analítica es una nueva forma que también genera eficiencia y eficacia en los procesos empresariales, sobre todo cuando las empresas están en crecimiento, ya que el Big Data permite mejorar mientras se ejercen acciones (Ekos 2016). Esta herramienta permite conocer la estructura interna y externa de la organización, es decir, a los trabajadores que la forman y a los clientes que son quienes necesitan que las empresas mejoren en torno a sus preferencias.

El nivel de productividad puede ser medida tanto cualitativamente como cuantitavamente (Allen y Meyer 1990), la medición cualitativa puede apegarse a conceptos corporativos básicos como la administración de las fuerzas de Michael Porter o el cumplimiento de reglamentos y estatutos internos. Por otro lado, para medir cuantitativamente la productividad se puede medir por tiempo de trabajo o por métodos financieros, donde el primero se basa en encontrar el resultado marginal por hora de trabajo y en el segundo, se busca encontrar el valor de la utilidad junto a su comparación con otros periodos (Prokopenko 1989).

Asimismo, existen empresas que no son afectadas por el uso de TI, ya sea por aspectos como la poca necesidad de TI al realizar un trabajo o el uso de métodos prácticos de organización que no requieren del uso de un software (Kalaignanam, Kushwaha y Steenkamp 2013).

\section{Gestión del conocimiento}

Davenport y Prusak (1998) definen al conocimiento como una mezcla fluida de la experiencia, valores, información contextual y visión experta que proporciona un marco teórico para evaluar e incorporar nuevas experiencias 
e información. Por otra parte, Prieto, Zambrano y Herrera (2014) indicaron que la gestión del conocimiento es la manera en que la información se procesa, con el fin de convertirse en una ventaja competitiva para las firmas consultoras.

La gestión del conocimiento, según Prieto (2005), es reconocer y administrar aquellas actividades y elementos de apoyo que resultan esenciales para atribuir a la organización que, al actuar como facilitadores, afectan al funcionamiento eficiente de los sistemas de aprendizaje y al valor de la organización en el mercado.

La suma del conocimiento y destrezas de las firmas determinan la habilidad de los nuevos emprendimientos para ofrecer una diversa gama de servicios con el fin de ganar aceptación en el mercado, mantenerse y lograr éxito financiero (Zahra y Bogner 1999). De esta forma, beneficiar continuamente a sus grupos de interés o stakeholders (Mayo y Lank 1994).

\section{Metodología}

Se empleó la metodología cualitativa bajo una lógica inductiva basada en el estudio de enunciados singulares que, en este caso, son la recopilación de datos en entrevistas a profundidad, ejecución de un grupo focal y cuestionarios semiestructurados. El diseño que se usó en la investigación es el de análisis del discurso, ya que permite sacar conclusiones relevantes de cada una de las personas (gerentes) entrevistadas.

La muestra fue no probabilística, dado que se realizó en función de las empresas consultoras registradas en la ciudad de Guayaquil que aceptaron su participación en la investigación. Las empresas presentan las siguientes particularidades: a) dos empresas de consultoría grandes, con más de 30 trabajadores, b) dos empresas de consultoría medianas, que poseen entre diez y 30 trabajadores, y c) dos empresas de consultoría pequeñas, que poseen menos de 10 trabajadores.

De un total de 10 empresas consultoras escogidas al azar, solo seis consultoras aceptaron participar en las entrevistas para la realización del presente artículo. 


\section{Desarrollo de la investigación}

Los servicios que destacan en la industria son la consultoría y el asesoramiento tecnológico, en donde la consultoría toma ventaja al ser una herramienta con amplia información compuesta de capital intelectual, convirtiéndose en un activo intangible valioso para las organizaciones (Liberona de la Fuente 2013).

Según Arzola y Mejías (2010), el estilo de aprendizaje y gestión del conocimiento son constructos que miden el desempeño y la productividad del sector de servicios y de la consultoría. La investigación tiene como objetivo el estudio de la relación que existe entre las empresas de servicios, específicamente consultorías de investigación de mercado y la relación que tienen al implementar las TI y gestionar el flujo de conocimiento dentro de la compañía (Alves de Almeida 2005). Se levantó información de un catastro de empresas consultoras en Guayaquil, con el fin de identificar a las principales empresas de consultoría; para ello se generaron 16 preguntas que permitieron mantener una matriz de información. Se obtuvieron datos que permitieron identificar diversos factores y, de los seis entrevistados, tres permitieron el uso de sus nombres en la investigación.

A continuación, la tabla 1 contiene las preguntas y respuestas de las seis empresas dedicadas a la consultoría de investigación de mercado, donde se visualiza cuál es la relación de la implementación de las TI y administración del conocimiento en las consultoras de Guayaquil.

Los entrevistados indicaron que conocen lo que son las TI; todos indicaron que utilizan TI en sus labores diarias. La pregunta donde se genera un contraste es en la tercera: ¿Qué tipo de tecnologías implementa o implementaría en su compañía?, dado que tres empresas planean implementar nuevas herramientas que les permitiría innovar en el mercado. Hubo una consultora que indicó no estar interesada en implementar las TI en sus procesos de mejora continua.

En la cuarta pregunta. ¿Qué tipo de conocimientos ha generado en su compañía la implementación de tecnologías de información?, se consultó cuáles son los cambios que ha generado la implementación de las TI; los entrevistados indicaron que las principales mejoras que se obtienen son la optimización de tiempo, disminución de errores e información interdeparta- 


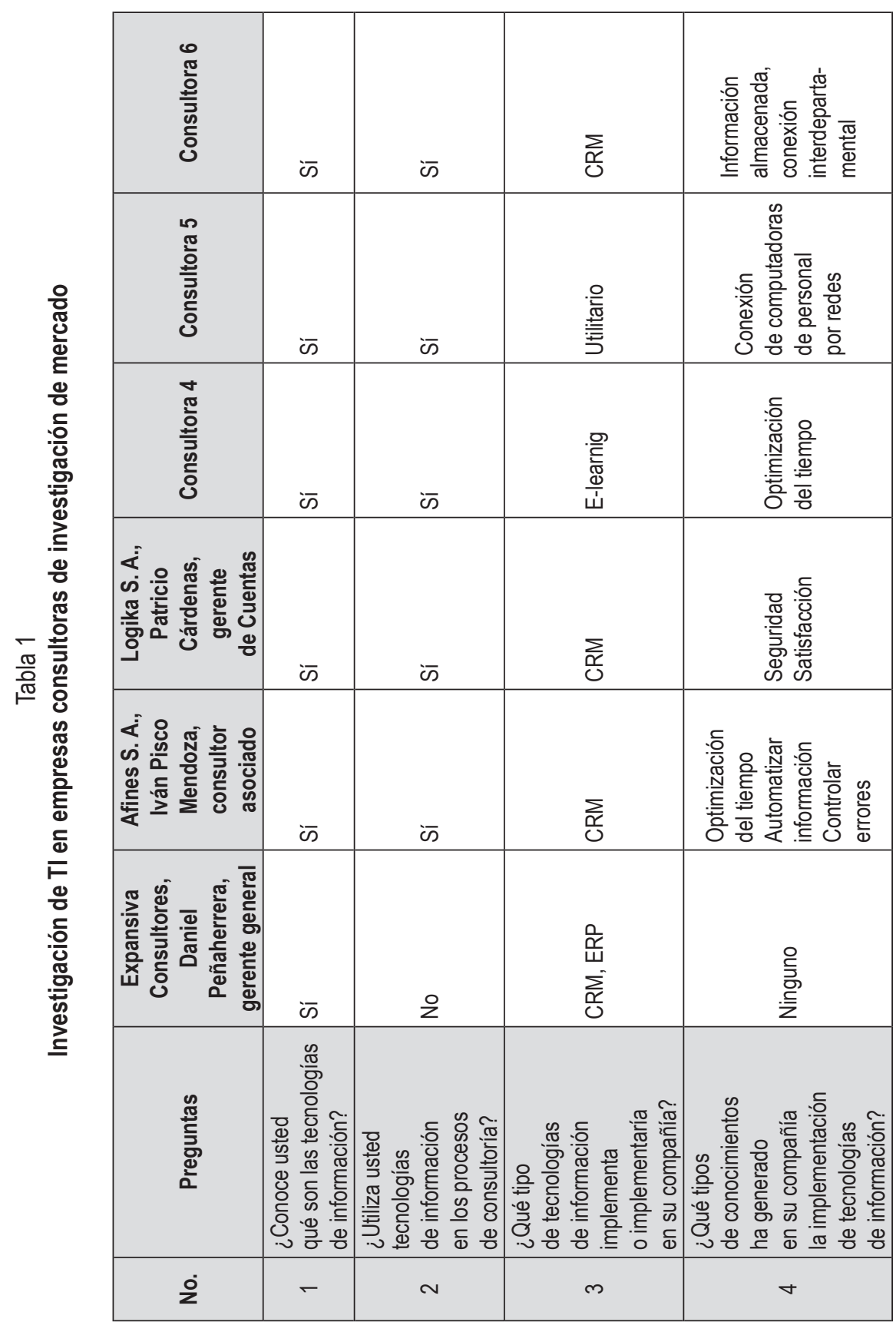


Implementación de tecnologías de información y administración del conocimiento

\begin{tabular}{|c|c|c|c|c|c|c|}
\hline 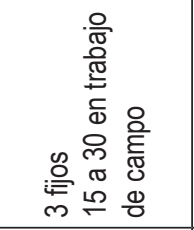 & is & 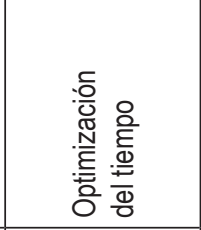 & 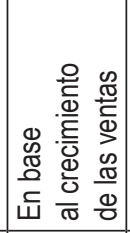 & 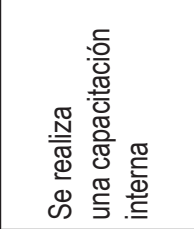 & ఉ & 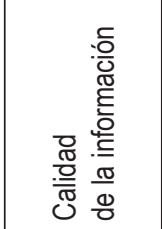 \\
\hline 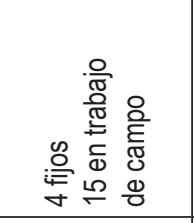 & क & 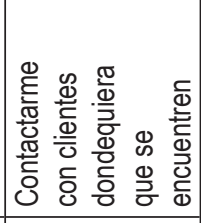 & 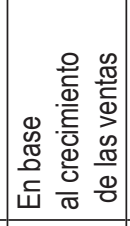 & 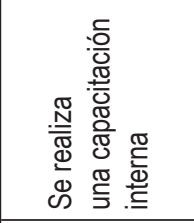 & $\bar{\omega}$ & 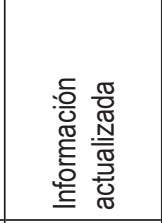 \\
\hline 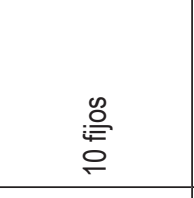 & ๘ & 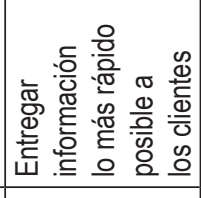 & 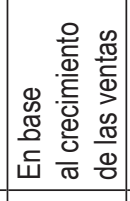 & 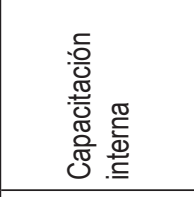 & ఉ & 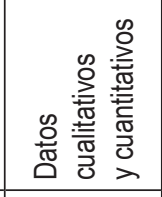 \\
\hline 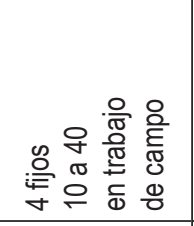 & ๘ & 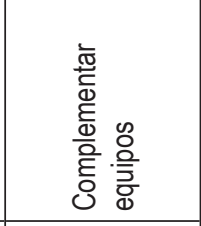 & 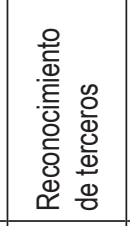 & 을 & $\frac{0}{2}$ & 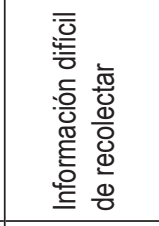 \\
\hline 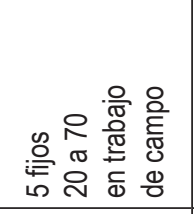 & ఉ & 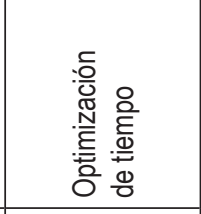 & 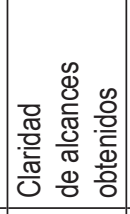 & 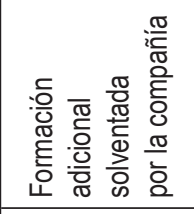 & ఉ & 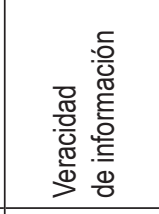 \\
\hline 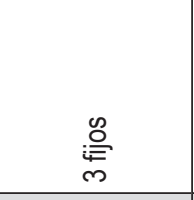 & ๘ & 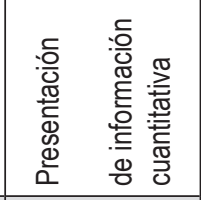 & 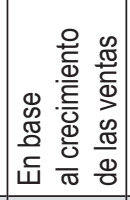 & 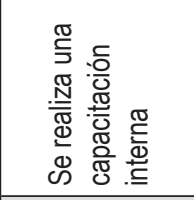 & 2 & 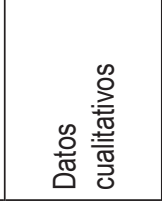 \\
\hline 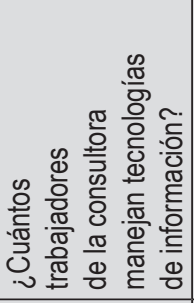 & 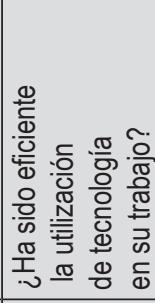 & 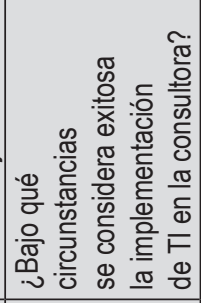 & 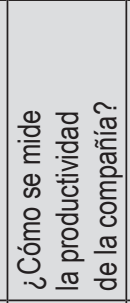 & 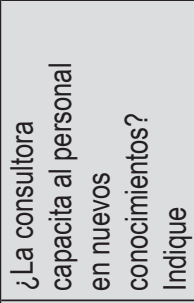 & 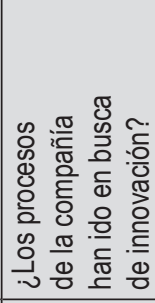 & 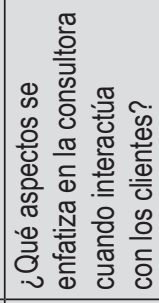 \\
\hline 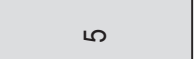 & 0 & 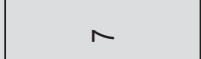 & $\infty$ & o) & 으 & $F$ \\
\hline
\end{tabular}


Danny Arévalo Avecillas y Carmen Padilla Lozano

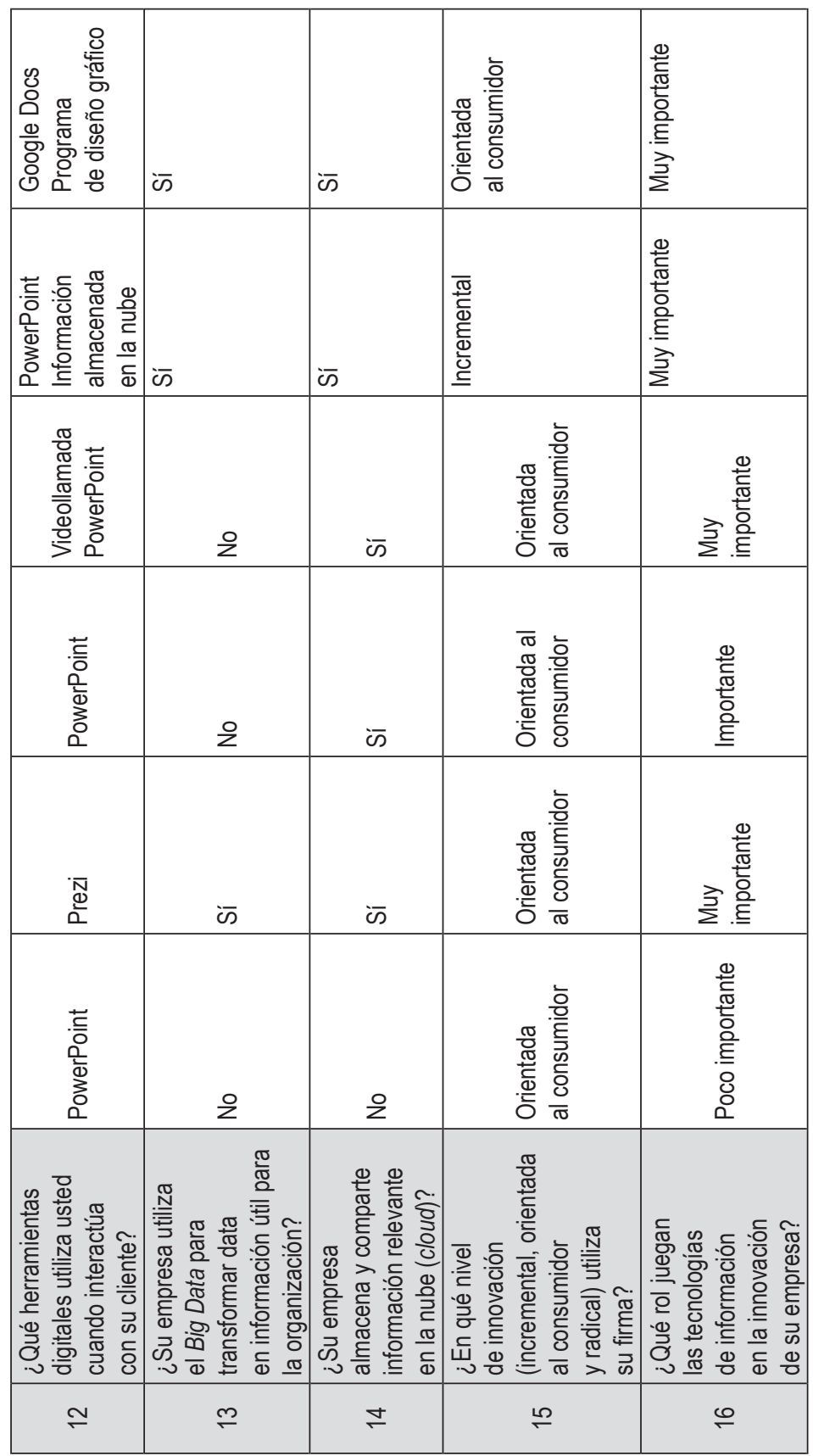

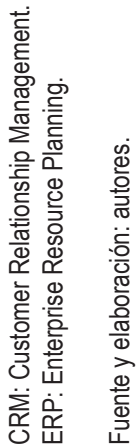


mental, lo que resulta en una eficiencia dentro del negocio. Las principales herramientas TI que utilizan las empresas son las relacionadas con ofimática, ya que en las empresas es la herramienta clave dentro de sus actividades.

Es importante acotar que el Big Data no es un tema muy conocido dentro de las firmas de consultoría, ya que se necesitan recursos tecnológicos de última tecnología y de talento humano especializado para poder transformar data en información relevante para las firmas consultoras. Sin embargo, el manejo de la información en la nube es una herramienta tecnológica muy utilizada por los gerentes en las consultoras, ya que es de mucha ayuda para interactuar e intercambiar información de relevancia con los clientes (Hwang, Kim y Lee 2015).

Si bien el uso de tecnologías de información y comunicación para el caso particular de una empresa puede representar esfuerzos e inversiones adicionales que pueden ir desde capacitación del personal hasta cambios radicales en las operaciones y en el aparato organizacional de la empresa (Hoyos Chaverra y Valencia Arias 2012), no debe constituirse en una razón para no incurrir en inversiones, ni de reducir el incentivo de utilización de este tipo de herramientas. Por consiguiente, se preguntó a los gerentes en qué nivel de innovación se encontraba su firma; la mayoría indicó que se encontraba en un nivel de innovación orientada a resolver los problemas de sus clientes. Solo hubo una consultora que indicó que su nivel de innovación era incremental, o sea utilizaba las TI para generar mejoras internas de los procesos de la firma.

Los hallazgos más relevantes de la presente investigación son: las empresas cuentan con conocimientos previos del manejo de la competencia en cuanto a informes, búsqueda de información, por lo que deciden implementar de manera progresiva las TI; luego de contar con procedimientos de trabajo sistematizados, buscan la manera de generar un cambio que les permita ser atraíbles dentro del servicio de consultoría; toda empresa que cuenta con factores de diferenciación destaca de su competencia. Además, se muestra la relación que existe dentro de las consultoras que al implementar las TI generan una ventaja competitiva frente a la competencia. Las firmas consultoras cuentan con conocimientos previos que permiten desarrollar las solicitudes de los clientes; sin embargo, el uso de las TI en el trabajo permite obtener un gran beneficio, desde el uso del computador para diseñar reportes hasta 
para la elaboración de un aplicativo móvil que permita obtener información actualizada de un cliente, el cual pueda acceder fácilmente a la información de relevancia.

La definición propuesta permite identificar que el conocimiento puede en cierta medida cuantificarse, ya que en la medida en que una organización es capaz de aprender algo nuevo, se constituye en un indicador del nivel de conocimientos (Alavi y Leidner 2001).

Darroch y McNaughton (2002) definieron la gestión del conocimiento en estudios previos como la función de la administración que crea o identifica el conocimiento, administra el flujo de conocimiento en la organización y asegura que dicho conocimiento sea usado efectiva y eficientemente para el beneficio a largo plazo de la organización. Dentro de su estudio indican que las innovaciones radicales provienen de una orientación tecnológica, dado

Figura 3

\section{La implementación de las TI y la administración del conocimiento para generar innovación}

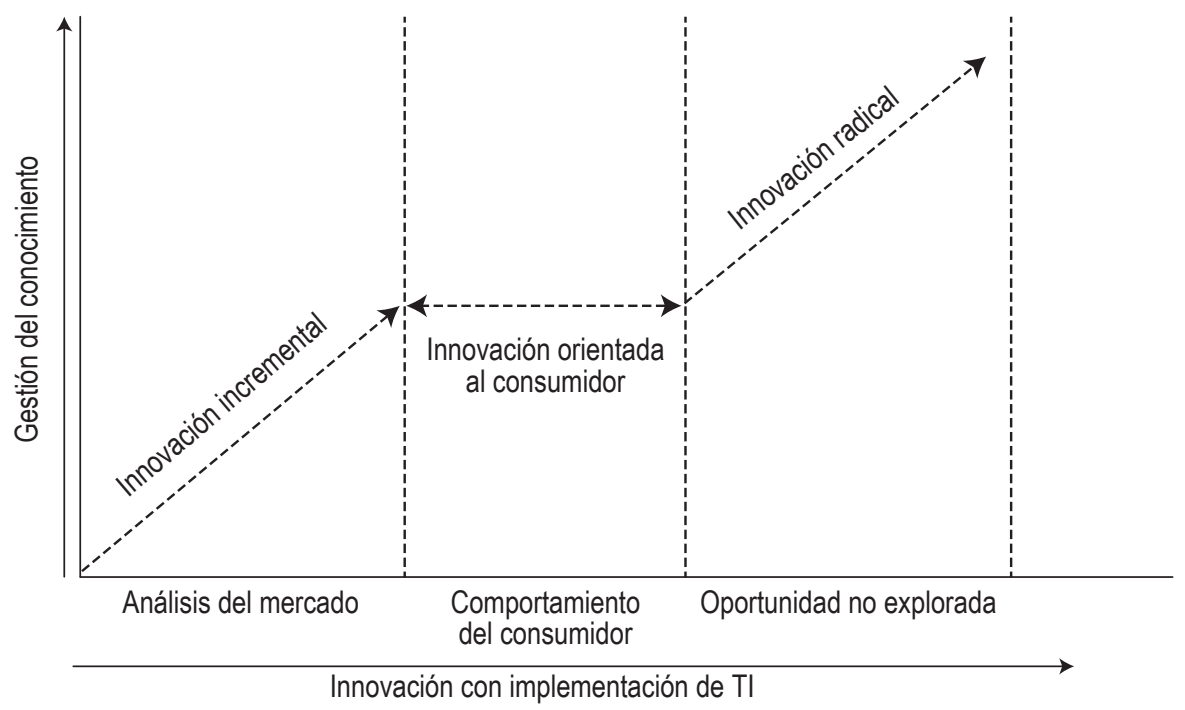

Fuente y elaboración: autores. 
que cambia lo que el consumidor requiere destruyendo a la competencia, por lo que consiguen transformar el negocio (Alavi y Leidner 2001). Por consiguiente, se preguntó a los gerentes en qué nivel de innovación se encontraba su firma; cinco gerentes indicaron que se encontraban en un nivel de innovación orientada a resolver los problemas de sus clientes. Como se mencionó anteriormente, solo hubo una consultora que indicó que su nivel de innovación era incremental, o sea utilizaba las TI para generar mejoras internas de los procesos de la firma.

La orientación hacia la innovación, que busca cambios en el consumidor, se mostró muy diferente a la innovación radical, en especial por no ser influenciadas por igual por el factor de orientación tecnológica, ya que consiste en la extensión o modificación de productos existentes, razón por la cual son consideradas como innovaciones empujadas por el mercado (Kohli y Jaworski 1990). Por otra parte, la innovación radical es considerada como destructora de la competencia; este tipo de innovación demanda prácticas administrativas diferentes y se originan de procesos científicos (Ettlie y Rubenstein 1987).

En la figura 3 se observa que la innovación radical es la que genera ventaja competitiva y es la que las consultoras deberían implementar.

A mayor búsqueda de recursos, investigación de oportunidades, mayor será la implementación de las TI, puesto que se buscará ser diferentes a los competidores.

La aparición de TI para la integración de procesos ha aportado a la transferencia de conocimientos (learning by doing) y la introducción de plataformas o software informáticos permite administrar de manera eficiente el conocimiento generado y en menor tiempo la información que se genere en los distintos departamentos de la empresa, teniendo como ejemplo de esto la administración de las relaciones con los clientes, Customer Relationship Management (CRM), y el almacenamiento de datos (Kalaignanam, Kushwaha y Steenkamp 2013).

Por último, se busca la innovación, ese factor diferencial que permite otorgar un producto o servicio de calidad a los clientes, mediante el uso de sistemas desarrollados en base a las necesidades de la compañía. Se puede eliminar la burocracia departamental dentro de las compañías, logrando que el personal trabaje en equipo y sistematizando la información para la toma 
Figura 4

\section{Etapas de incorporación de las TI en empresas}

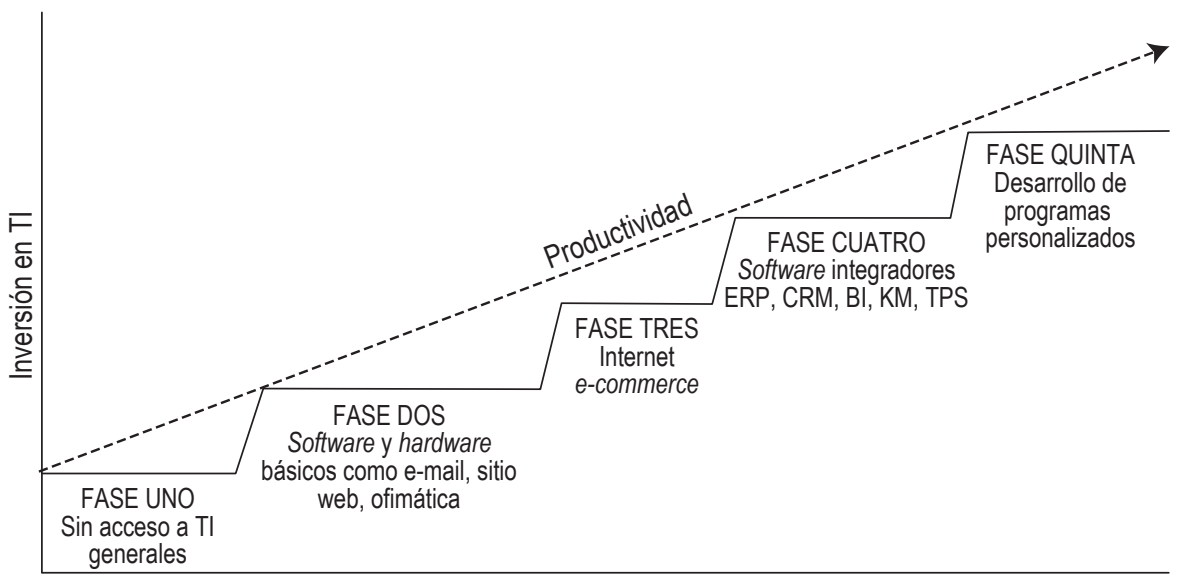

Fases de la incorporación de TI

Fuente y elaboración: autores.

de decisiones de manera efectiva. En efecto, se puede visualizar el impacto positivo que se tiene como oportunidad al implementar TI.

Para Bosworth y Collins (2008), los servicios pueden ser un motor de crecimiento de exportación para algunos países. Las empresas consultoras tienen el objetivo de actualizar e incrementar el conocimiento del proyecto, lo que permite una experiencia acumulada para futura toma de decisiones o implementación de nuevos proyectos (Allen y Meyer 1990).

Finalmente, se preguntó a los gerentes si las TI juegan un papel importante en la mejora de procesos e innovación de la firma para generar nuevos servicios. Las respuestas fueron muy variadas, tres consultoras indicaron que las TI son muy importantes para generar innovación en sus procesos internos y las demás indicaron que se requiere de muchos recursos tecnológicos, humanos y financieros para alcanzar esa meta y que las empresas ecuatorianas no están lista para mejorar sus procesos de innovación apoyados en las diversas herramientas tecnológicas. 
Figura 5

Mapa cognitivo de las $\mathrm{Tl}$ en consultoras

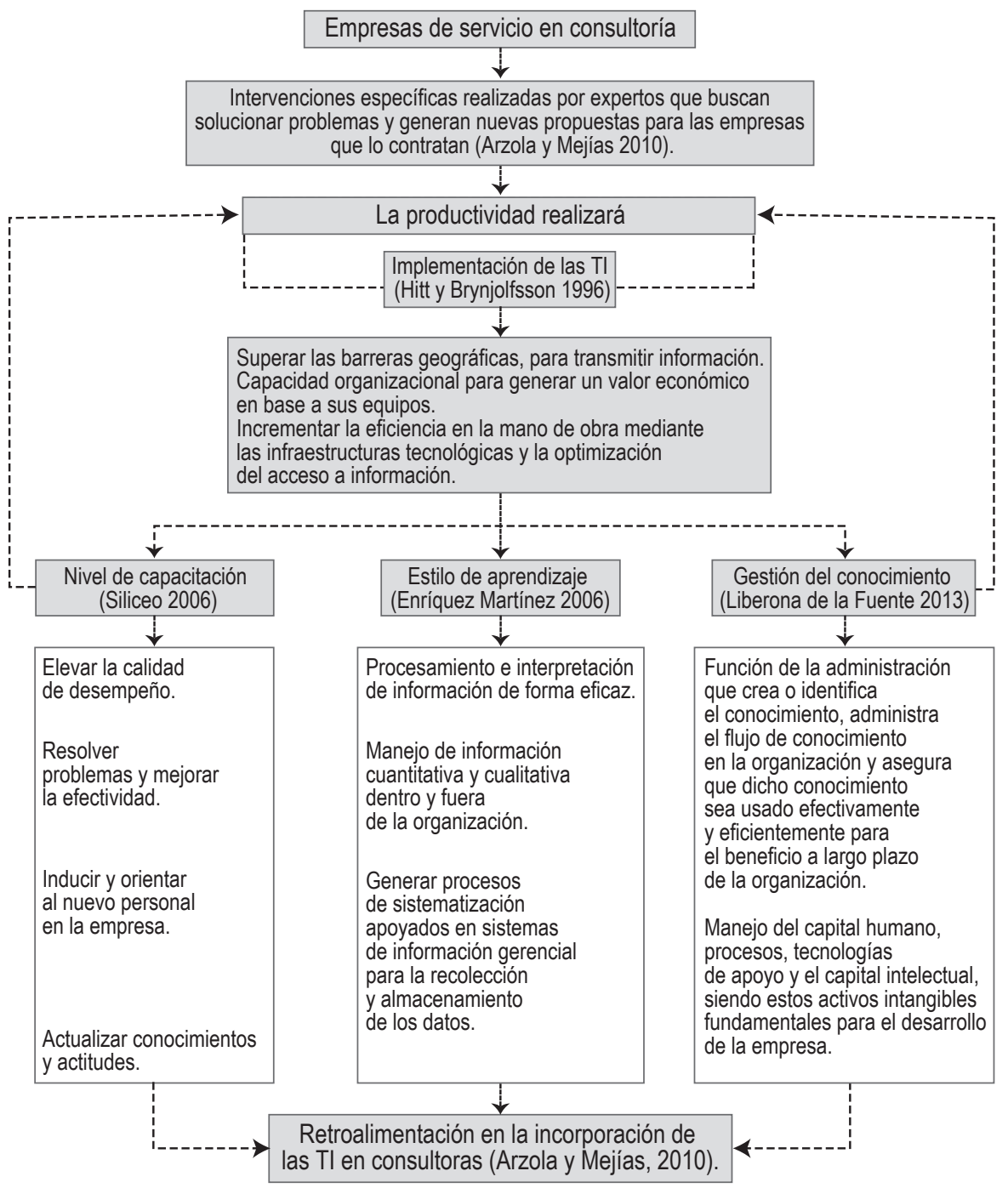

Fuente: según referencias en la figura.

Elaboración propia. 


\section{Conclusiones}

El reconocimiento del grado de importancia que tienen las TI en las empresas consultoras situadas en Guayaquil nos permiten inferir que las TI no son solamente un grupo de herramientas de ofimática sino toda aquella tecnología capaz de desarrollar dinamismo en la empresa y en el mercado. En efecto, como se expresa en la figura 5, se subdividen en componentes de los cuales se busca la mejora continua. El manejo de las TI son útiles en las actividades diarias de las firmas. Como enseñanza primordial nos queda que, si implementamos TI en las empresas, acompañadas de un nivel óptimo de capacitación, un correcto estilo de aprendizaje organizacional y una buena gestión del conocimiento, llegaremos a ser más productivos y alcanzar un rendimiento óptimo, que nos permita diferenciarnos del mercado y posicionarnos a mediano y largo plazo de forma eficiente, alcanzando cada vez más la anhelada ventaja competitiva.

Se observa en esta investigación que las seis empresas encuestadas utilizan TI en sus actividades, ya sea para obtener información cuantitativa o cualitativa. Se evidenció la utilización de ofimática y Microsoft Office, mientras que tres de los encuestados prefirió contratar un desarrollador de software que le permita evolucionar acorde al mercado.

Es necesario establecer los elementos y criterios que relacionan las TI y la productividad. Esto toma relevancia conociendo que existen sesgos y subjetividad por parte del empresario en general respecto a estas decisiones de inversión por la recuperación de inversión en el menor tiempo posible (corto plazo). Se conocen esfuerzos gubernamentales para generar capacitaciones y cursos orientados a proporcionar información de mercado; pocas son las empresas que consideran una inversión considerable en implementación de TI dentro del sector de servicios de consultoría.

Las empresas demandantes de los servicios de investigación de mercados se encuentran sesgadas por actores subjetivos propios del entorno empresarial ecuatoriano, los que inciden negativamente en la aceptación del sector de servicios en consultoría y gestión empresarial.

Las empresas utilizan como herramienta de innovación la información almacenada en la nube, desarrollo de infografías que permitan el dinamismo con el cliente y automatización de procesos. El consultor tiene la responsabi- 
lidad de construir una red de aprendizaje de alta experiencia tanto en conocimientos teóricos como en la práctica del desenvolvimiento de las industrias. Por otro lado, el entorno empresarial ha cambiado significativamente. La convergencia de múltiples tecnologías de punta, combinada con una competencia sin precedentes y tiempos económicos complejos, exige una nueva estrategia de tecnología de la empresa que cambie fundamentalmente la forma en que estos sistemas se desarrollan, mejoran y mantienen la TI en todos sus procesos internos y externos. La innovación de cinco empresas en consultoría es orientada al consumidor y una presenta una innovación radical.

\section{Recomendaciones}

El estudio proporciona beneficios en la productividad por las aplicaciones de TI en empresas que realizan servicios de consultorías. Las consultoras deben buscar innovar en el mercado para tener un factor diferenciador contra la competencia. Se recomienda a las empresas consultoras el desarrollo de un software aplicado a sus necesidades y al mercado, y capacitar al personal en el manejo de las TI para que aumenten la eficacia de proyectos de los clientes. Actualizarse en las nuevas TI es vital para este giro de negocio, puesto que no debe contar con conocimientos obsoletos, los cuales no permitan ofertar productos diferenciadores. Los investigadores de mercado comparten sus conocimientos tanto con los trabajadores como con los clientes.

Los resultados obtenidos podrán ser útiles en nuevas investigaciones, las cuales deberán enfocarse en las oportunidades del mercado en firmas consultoras, comparación con diferentes industrias, elaboración de estudios longitudinales donde se pueda observar el impacto de la productividad frente a la competencia e imagen de las firmas consultoras en el mercado. La productividad dentro de las compañías es generada por otros factores diferentes, por lo que se recomienda realizar un análisis de factores en las compañías que ofertan el servicio de consultoría. 


\section{Referencias}

Alavi, Maryam, y Dorothy Leidner. 2001. "Knowledge Management and Knowledge Managment Systems: Conceptual Foundations and Research Issues”. MIS Quarterly 25, No. 1 (marzo): 107-136.

Allen, Natalie, y John P. Meyer. 1990. "The Measurement and Antecedents of Affective, Continuance and Normative Commitment to the Organization”. Journal of Occupational Psychology 63, No. 1: 1-18.

Alves de Almeida, María do Rosário. 2005. "La dirección de los recursos humanos y las nuevas tecnologías de la información y la comunicación”. Tesis doctoral en Administración de Empresas y Comercialización e Investigación de Mercados. Universidad de Sevilla, Sevilla.

Aral Sinan, Erik Brynjolfsson, y Lynn Wu. 2012. "Three-Way Complementarities: Performance Pay, Human Resource Analytics, and Information Technology". Management Science 58, No. 5: 913-931.

Arzola, Minerva, y Agustín Mejías. 2010. "Morfología del aprendizaje y la gestión del conocimiento del sector servicios de consultoría”. Ingeniería Industrial 9, No. 1: 37-51.

Bartolomé, Antonio. 1995. "Preparando para un nuevo modo de conocer". Revista Electrónica de Tecnología Educativa, No. 4: 1-12.

Bayo-Moriones, Alberto, Margarita Billón y Fernando Lera-López. 2013. "Perceived Performance Effects of ICT in Manufacturing SMEs". Industrial Management \& Data Systems 113, No. 1: 117-135.

Bosworth, Barry, y Susan Collins. 2008. "Accounting for Growth: Comparing China and India”. Journal of Economic Perspectives 22, No. 1: 45-66.

Botella Arbona, y otros. 2010. “Avances en los tratamientos psicológicos: la utilización de las nuevas tecnologías de la información y la comunicación”. Anuario de Psicología 40, No. 2: 155-170.

Cañellas, Aries. 2006. "Impacto de las TIC en la educación: un acercamiento desde el punto de vista de las funciones de la educación". Revista de nuevas tecnologías y sociedad, No. 43: $12-16$.

Cimoli, Mario, y Nelson Correa. 2010. ICT, Learning and Growth: An Evolutionary Perspective. Documento de trabajo. CEPAL / UNDP. Consulta: diciembre de 2016. 〈https://ideas. repec.org/h/elg/eechap/13996_7.html.

Darroch, Jenny, y Rod McNaughton. 2002. "Examining the Link Between Knowledge Management Practices and Types of Innovation”. Journal of Intellectual Capital 28, No. 1: 210-222.

Davenport, Thomas, y Prusak Lawrence. 1998. Working Knowledge: How Organizations Manage What They Know. Cambridge: Harvard Business School Press. 
Implementación de tecnologías de información y administración del conocimiento

Devaraj, Sarv, y Rajiv Kohli. 2003. "Performance Impacts of Information Technology: Is Actual Usage the Missing Link?”. Management Science 49, No. 3: 273-289.

Enríquez Martínez, Álvaro. 2006. "La significación en la cultura: concepto base para el aprendizaje organizacional”. Universitas Psychologica 6, No. 1: 155-162.

Ettlie, John, y Albert Rubenstein. 1987. "Firm Size and Product Innovation". Journal of Product Innovation Management 4, No. 2: 77-165.

Francois, Joseph, y Bernard Hoekman. 2010. "Services Trade and Policy". Journal of Economic Literature 48, No. 3: 642-692.

Freeman, Christopher. 1974. La teoría económica de la innovación industrial. Madrid: Alianza.

Hitt, Lorin, y Erick Brynjolfsson. 1996. "Productivity, Business Profitability and Consumer Surplus: Three Different Measures of Information Technology Value”. MIS Quarterly 20, No. 2: 121-142.

Hoyos Chaverra, Jonathan, y Alejandro Valencia Arias. 2012. "El papel de las TIC en el entorno organizacional de las PYMES”. Trilogía, No. 7: 105-122.

Hwang, Jong-Sung, Sung Hyun Kim y Ho Lee. 2015. "Breaking the Myths of the IT Productivity". KSII Transactions on Internet and Information Systems 9, No. 1: 466-482.

Instituto Nacional de Estadística y Censos (INEC). 2010. Censo Nacional Económico. Consulta: enero de 2016. 〈http://www.ecuadorencifras.gob.ec/〉.

---. 2014. Censo Nacional Económico. Consulta: noviembre de 2016. 〈http://www.ecuador encifras.gob.ec/>.

Instituto Nacional de Estadística e Informática (INEI). 2010. Clasificación industrial internacional uniforme de todas las actividades económicas. Revisión 4. Lima: INEI.

Kalaignanam, Kartik, Tarun Kushwaha y Jan-Bemedict Steenkamp. 2013. "The Effect of CRM Outsourcing on Shareholder Value: A Contingency Perspective". Management Sciences 59, No. 3: 748-769.

Kantis, Hugo. 1996. Inercia e innovación en las conductas estratégicas de las pymes argentinas: elementos conceptuales y evidencias empíricas. Buenos Aires: CEPAL.

Karantinou, Kalipso, y Margaret K. Hogg. 2001. "Exploring Relationship Management in Professional Services: A Study of Management Consultancy”. Journal of Marketing Management 17, No. 4: 263-286.

Kholi, Ajay, y Bernard Jaworski. 1990. "Market Orientation: The Construct, Research Propositions, and Managerial Implications”. Marketing Science Institute 90, No. 113: 1-40.

Kubr, Milan. 2002. Management Consulting: A Guide to the Profession. Ginebra: OIT.

Liberona de la Fuente, Darío. 2013. “Análisis de las estrategias organizacionales y tecnológicas para implementar programas de gestión del conocimiento en empresas chilenas". Tesis doctoral en Gestión Avanzada de Empresas. Universidad de Lérida, Lérida.

López, Esteban, Claudia Sepúlveda y Henry Antonio Arenas. 2010. "La consultoría de gestión humana en empresas medianas”. Estudios Gerenciales 26, No. 114: 10-18. 
Maister, David, Charles Green y Robert Galdford. 2000. The Trusted Advisor. Nueva York: The Free Press.

Maldonado Guzmán, Gonzalo, y otros. 2010. "La influencia de las TIC en el rendimiento de la PyME de Aguascalientes". Investigación y Ciencia, No. 47: 57-65.

Mayo, Andrew, y Elizabeth Lank. 1994. Las organizaciones que aprenden. Barcelona: Gestión 2000.

Nachum, Lilach. 1999. "Measuring the Productivity of Professional Services: A Case Study on Swedish Management Consulting Firms". Documento de trabajo, ESRC Center for Business Research, University of Cambridge, Cambridge. Consulta: mayo de 2015. «ttp:// www.reserachgate.net/publuication4924948 .

Nolan, Richard, y Warren McFarlan. 2005. "Information Technology and the Board of Directors. Harvard Business Review 83, No. 10: 96-107.

Prieto, Ana Teresa, Calixto Zambrano y Norka Herrera. 2014. "Gestión del conocimiento en las empresas de servicios a pozos petroleros". Revista de Ciencias Sociales 20, No. 1: 56-70.

Prieto, Isabel. 2005. Gestión del conocimiento para el desarrollo de la capacidad de aprendizaje en las organizaciones. Valladolid: Universidad de Valladolid.

Prokopenko, Joseph. 1989. La gestión de la productividad: manual práctico. Ginebra: OIT.

Revista Ekos. 2013. "Perspectiva general". Ekos Negocios, No. 72: 168-178. Consulta: abril de 2016: 102. 〈https://issuu.com/ekosnegocios/docs/ekos224_baja〉.

---. 2014. "Ranking 1.000 Ecuador 2016". Ekos Negocios, No. 70. Consulta: abril de 2016: 102. 〈http://www.ekosnegocios.com/negocios/verArticuloContenido.aspx?idArt=7919〉.

---. 2016. "Cuando el Cloud es tangible en los negocios". Ekos Negocios. No. 76. Consulta: enero de 2016. 〈https://issuu.com/ekosnegocios/docs/267_ca0b2d60c40f6c〉.

Vistazo. 2013. "100 marcas Top of Mind". Vistazo, No. 65: 118-119. Consulta: abril de 2016. 〈http://vistazo.com/seccion/edicion-impresa/especial-100-marcas-top-mind〉.

Siliceo. Alfonso. 2006. Capacitación y desarrollo personal. Ciudad de México: Limusa.

Tung, Lai Lai, y Olaf Rieck. 2005. "Adoption of Electronic Government Services Among Business Organizations in Singapore". The Journal of Strategic Information Systems 14, No. 4: 417-440.

Wade, Michael, y John Hulland. 2004. "The Resource-Based View and Information Systems Research: Review, Extension and Suggestions for Future Research". Management Information Systems Quarterly 28, No. 1: 25-32.

World Economic Forum (WEF). 2014. Networked Readiness Index Ranking. Consulta: enero de 2016. «ttp://reports.weforum.org/global-information-technology-report-2016/network ed-readiness-index/s.

Zahra, Shaker, y William Bogner. 1999. "Technology Strategy and Software New Adventure's Performance: Exploring the Moderating Effect of the Competitive Enviroment". Journal of Business Venturing, No. 15: 135-173. 